\title{
On Apparent Activation Energy of Structure Sensitive Heterogeneous Catalytic Reactions
}

\author{
Dmitry Yu. Murzin ${ }^{1}(1)$
}

Received: 26 February 2019 / Accepted: 21 March 2019 / Published online: 3 April 2019

(C) The Author(s) 2019

\section{Abstract}

The notion of apparent activation energy is discussed in relation to structure sensitivity of complex heterogeneous catalytic reactions comprising several steps. The cases of nonuniform surfaces (intrinsic and induced) are considered, showing that the apparent activation energy depends on the cluster size and surface coverage. The theoretical analysis of the apparent activation energy was also extended for the two step sequence with deactivating catalyst by poisoning or when coking occurs as a result of the reactant interactions with an intermediate.

\section{Graphical Abstract}

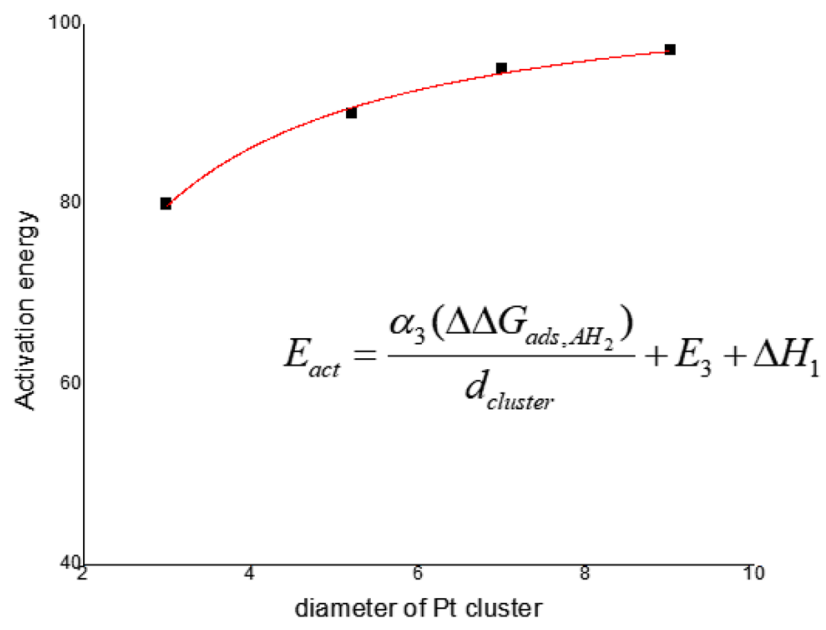

Keywords Structure sensitivity $\cdot$ Apparent activation energy $\cdot$ Cluster size $\cdot$ Deactivation

\section{Introduction}

Kinetic analysis of complex non-catalytic and catalytic organic reactions is more and more utilized in academia and industry [1-5]. Such analysis became a routine part of chemical reaction engineering [6-10], where rather complex

Dmitry Yu. Murzin

dmurzin@abo.fi

1 Johan Gadolin Process Chemistry Centre, Åbo Akademi University, Turku/Åbo, Finland mechanisms are rigorously treated using numerical data fitting.

An opposite, but understandable, trend, stemming from a desire to diminish complexity, is to express the rate $(r)$ in a simplistic way with some global reaction parameters, such as apparent orders in reactants and catalysts or apparent activation energy

$r=k_{0} e^{-E_{\text {act }} / R T} C_{\text {subs }}^{m_{i}} C_{\text {catal }}^{n}$

where $r$ is the rate, concentration of substrate $i, \mathrm{n}^{-}$- reaction order in this compound, $\mathrm{E}_{\mathrm{act}}$-apparent activation energy. 
Utilization of simple approaches to interpret kinetic data can be a valuable tool for the study of reaction mechanisms. For example, reaction orders in the substrates can point out on the mechanistic aspects of a particular compound involvement in the catalytic cycle [11-15]. Often the reaction orders in hydrogen and oxygen in respective hydrogenation and oxidation reaction equal to 0.5 are taken as an argument in favour of dissociative adsorption, while a reaction order in catalysts below unity [5] is associated with formation of dimeric catalytic species [13-15].

While fractional orders almost immediately point out on complex reaction mechanisms and are viewed in this way by both experts and non-experts in kinetics, interpretation of the activation energy is less straightforward, even if it was recognized long time ago that apparent activation energy for a heterogeneous catalytic reaction depends on the heat of adsorption of reactants [18].

Rigorous analysis of apparent activation energy was described in [19] for the two-step mechanism with two kinetically significant steps $[6,16,17]$, and one most abundant surface intermediate

$$
\begin{array}{r}
1 . \mathrm{Z}+\mathrm{A} \leftrightarrow \mathrm{ZI}+\mathrm{C} \\
\frac{2 . \mathrm{ZI}+\mathrm{B} \leftrightarrow \mathrm{Z}+\mathrm{D}}{\mathrm{A}+\mathrm{B} \leftrightarrow \mathrm{C}+\mathrm{D}}
\end{array}
$$

In Eq. (2) $A, B$ are reactants, $C$, and $D$ are products, $Z$ is the surface site and $I$ is an adsorbed intermediate. The expression for turnover frequency for this mechanism is given by [16].

$v(d)=\frac{k_{1} k_{2} P_{A} P_{B}-k_{-1} k_{-2} P_{C} P_{D}}{k_{1} P_{A}+k_{-2} P_{D}+k_{2} P_{B}+k_{-1} P_{C}}$

Considering that

$E_{\mathrm{a}, \mathrm{app}}=-R \frac{\partial \ln r}{\partial(1 / T)}$

for a reaction with both irreversible steps considered in [19] the apparent activation energy can be calculated as

$E_{\mathrm{a}, \mathrm{app}}=E_{\mathrm{a}, 1}+E_{\mathrm{a}, 2}-\frac{k_{1} P_{\mathrm{A}}}{k_{1} P_{A}+k_{2} P_{B}} E_{\mathrm{a}, 1}-\frac{k_{2} P_{B}}{k_{1} P_{A}+k_{2} P_{B}} E_{\mathrm{a}, 2}$

In the classical treatment of [19] dependence of the structure sensitivity $[20,21]$ on apparent activation energy was not considered. Therefore, the main focus in the current work is on analyzing the apparent activation energy and overall temperature dependence for representative reaction mechanisms, when the cluster size dependence is incorporated in the rate constants. In the previous studies of the author [22-24] derivation of the rate equations has been already reported for the two step sequences on uniform and nonuniform surfaces as well as for the Langmuir-Hinshelwood type of mechanism. These cases will be considered in this work.

\section{Apparent Activation Energy for Two-Step Sequence on Nonideal Surfaces}

\subsection{Structure Sensitivity}

The treatment above (Eq. 5) assumed ideal surfaces in a sense that all surface sites were considered to be the same or lateral interactions were neglected. In other words the reaction is viewed as structure insensitive, which is often not the case.

A discrete approach accounting for different sites considers TOF as a sum over contributions of terraces (t), steps (s) and corners (c) [25-27] with the corresponding geometrical factors in the following way:

$v(d)=r_{\text {terraces }} f_{\text {terraces }}+r_{\text {corners }} f_{\text {corners }}+r_{\text {edges }} f_{\text {edges }}$

where $f_{\text {terraces }}, f_{\text {edges }}$ and $f_{\text {corners }}$ denote the fractions of these surface sites and can be calculated knowing relative fractions of terraces, edges and corners for a particular cluster geometry (cube, etc.) similar to [28], in which the first step in Eq. (2) was considered reversible

$$
\begin{aligned}
v(d)= & \frac{k_{1}^{c} k_{2}^{c} P_{A} P_{B}}{k_{1}^{c} P_{A}+k_{2}^{c} P_{B}+k_{-1}^{c} P_{C}} f_{\text {corners }} \\
& +\frac{k_{1}^{e} k_{2}^{e} P_{A} P_{B}}{k_{1}^{e} P_{A}+k_{2}^{e} P_{B}+k_{-1}^{e} P_{C}} f_{\text {edges }} \\
& +\frac{k_{1}^{t} k_{2}^{t} P_{A} P_{B}}{k_{1}^{t} P_{A}+k_{2}^{t} P_{B}+k_{-1}^{t} P_{C}} f_{\text {terraces }}
\end{aligned}
$$

Analysis of the temperature dependence for Eq. (7) is not straightforward as the rate constants for different sites can, in principle, have different activation energy.

An alternative approach is to consider a continuous dependence of the rate constant on the cluster size [22] giving an expression of TOF for the two-step sequence

$v(d)=\frac{\left(k_{1} k_{2} P_{A} P_{B}-k_{-1} k_{-2} P_{C} P_{D}\right) e^{(1-2 \alpha) \chi / d_{\text {cluster }}}}{\left(k_{1} P_{A}+k_{-2} P_{D}\right) e^{-\alpha \chi / d_{\text {cluster }}}+\left(k_{2} P_{B}+k_{-1} P_{C}\right) e^{(1-\alpha) \chi / d_{\text {cluster }}}}$

where $\alpha$ is the Polanyi parameter, $d_{\text {cluster }}$ is given in $\mathrm{nm}$ and $\chi$ reflects the difference between Gibbs energy of adsorption on edges and terraces:

$\chi=\frac{\left(\Delta G_{a d s, e d g e s}-\Delta G_{a d s, t e r r a c e s}\right)}{R T}$

Adsorption on corners was not explicitly addressed in [22], due to a low fraction of corners in typical 
heterogeneous catalysts with clusters having the size of few nanometers. Analysis in [22] was thus based on the following considerations

$$
\begin{aligned}
\Delta G_{\text {ads }} & =\Delta G_{\text {ads,terraces }}\left(1-f_{\text {edges }}\right)+\Delta G_{\text {ads,edges }} f_{\text {edges }} \\
& =\Delta G_{\text {ads,terraces }}+f_{\text {edges }}\left(\Delta G_{\text {ads,edges }}-\Delta G_{\text {ads,terraces }}\right)
\end{aligned}
$$

where $\Delta G_{\text {ads,terraces }}$ and $\Delta G_{\text {ads,edges }}$ correspond respectively to adsorption on terraces and edges.

In a particular case of the two step sequence with the second step being irreversible

$v(d)=\frac{k_{1} k_{2} P_{A} P_{B}}{k_{1} P_{A}+k_{2} P_{B}+k_{-1} P_{C}}$

Equation (11) can be rearranged using expressions for pre-exponential factors from the transition state theory [29]

$v(d)=\frac{a_{1} e^{\frac{(1-\alpha)\left(\Delta \Delta G_{\text {ads }}\right) / d_{\text {cluser }}-E_{2}}{R T}}}{1+a_{3}^{-1} e^{\left(-\left(E_{2}-E_{1}\right)+\Delta \Delta G_{\text {ads }} / d_{\text {cluser }}\right) / R T}+\left(a_{2} / a_{3}\right) e^{\left(\Delta \Delta G_{\text {ads }} / d_{\text {cluser }}-\Delta H_{1}\right) / R T}}$

with $\Delta \Delta G_{a d s}=\left(\Delta G_{a d s, e d g e s}-\Delta G_{a d s, t e r r a c e s}\right)$. In Eq. (12) $a_{1}$-a are lumped parameters, related to reactions on terraces, and include besides partition functions of reactants $\left(q_{B}, q_{A}\right)$, vacant sites, surface intermediate $\left(q_{Z}, q_{Z I}\right)$ and transition states $q_{T_{1}}^{*}, q_{T_{2}}^{*}$, also partial pressures of the reactants in the following way

$a_{1}=\frac{k_{B} T}{h} \frac{q_{T_{2}}^{*}}{q_{B} q_{Z I}} P_{B} ; a_{2}=\frac{q_{T_{1}}^{*}}{q_{T_{2}}^{*}} \frac{q_{B}}{q_{C}} \frac{P_{C}}{P_{B}} ; a_{3}=\frac{q_{T_{1}}^{*}}{q_{T_{2}}^{*}} \frac{q_{B} q_{Z I}}{q_{A} q_{Z}} \frac{P_{A}}{P_{B}}$.

Apparently, when there is a negligible difference between Gibbs energy of adsorption on edges and terraces $\Delta \Delta G_{a d s} \approx 0$, Eq. (12) is reduced to a special case when the reaction is structure insensitive
When $1>>a_{3}^{-1} e^{-\left(E_{2}-E_{1}\right) / R T}+a_{2} / a_{3} e^{-\Delta H_{1} / / R T}$ the activation energy is equal to the activation energy of the second step. Otherwise, the value of activation energy can vary depending on the relative contribution of terms in the denominator and, more specifically, values of concentrations and partition functions. In a general case of the structure sensitive reactions the apparent activation energy depends not only on the activation energy of the second step $E_{2}$, but also on the cluster size. This can be illustrated by considering that $E_{a c t}=R T^{2}(\partial \ln r / \partial T)$ thus giving an overall expression for the activation energy

$$
\begin{aligned}
& E_{\text {act }}=E_{2}-(1-\alpha)\left(\Delta \Delta G_{a d s}\right) / d_{\text {cluster }}+ \\
& +\frac{\left(E_{2}-E_{1}-\Delta \Delta G_{\text {ads }} / d_{\text {chster }}\right) a_{3}^{-1} e^{\left(-\left(E_{2}-E_{1}\right)+\Delta \Delta G_{\text {ads }} / d_{\text {clusser }}\right) / R T}}{1+a_{3}^{-1} e^{\left(-\left(E_{2}-E_{1}\right)+\Delta \Delta G_{\text {ads }} / d_{\text {cluster }} / R T\right.}+\left(a_{2} / a_{3}\right) e^{\left(\Delta \Delta G_{\text {ads }} / d_{\text {cluster }}-\Delta H_{1}\right) / R T}}- \\
& -\frac{\left(\Delta \Delta G_{a d s} / d_{\text {cluster }}-\Delta H_{1}\right)\left(a_{2} / a_{3}\right) e^{\left(\Delta \Delta G_{a d s} / d_{\text {cluster }}-\Delta H_{1}\right) / R T}}{1+a_{3}^{-1} e^{\left(-\left(E_{2}-E_{1}\right)+\Delta \Delta G_{\text {ads }} / d_{\text {cluster }}\right) / R T}+\left(a_{2} / a_{3}\right) e^{\left(\Delta \Delta G_{a d s} / d_{\text {cluster }}-\Delta H_{1}\right) / R T}}
\end{aligned}
$$

Equation (16) illustrates that the apparent activation energy has a complex dependence on the cluster size. For a particular case of the reaction mechanism when only the second step determines the overall rate, Eq. (16) can be essentially simplified giving

$E_{\text {act }}=E_{2}-(1-\alpha)\left(\Delta \Delta G_{\text {ads }}\right) / d_{\text {cluster }}$.

It follows from Eq. (17) that the activation energy can either increase or decrease as a function of the cluster size depending on which types of sites the Gibbs energy of adsorption is larger.

$k_{a p p}^{\prime} e^{-E_{a c t} / R T}=\frac{a_{1} e^{-E_{2} / R T}}{1+\frac{e^{-E_{2} / R T}+a_{2} e^{-E_{-1} / R T}}{a_{3} e^{-E_{1} / R T}}}=\frac{a_{1} e^{-E_{2} / R T}}{1+a_{3}^{-1} e^{-\left(E_{2}-E_{1}\right) / R T}+\left(a_{2} / a_{3}\right) e^{-\left(E_{-1}-E_{1}\right) / R T}}$.

Analysis of apparent activation energy utilizing Eq. (14) can be done by comparing terms in the denominator of the right- hand side of Eq. (14). The difference between the activation energy of the first step in the reverse and forward directions is equal to the enthalpy of this step, defined as $E_{-1}-E_{1}$, thus Eq. (14) takes the form

$k_{a p p}^{\prime} e^{-E_{a c t} / R T}=\frac{a_{1} e^{-E_{2} / R T}}{1+a_{3}^{-1} e^{-\left(E_{2}-E_{1}\right) / R T}+\left(a_{2} / a_{3}\right) e^{-\Delta H_{1} / R T}}$

\subsection{Lateral Interactions}

For the analysis of the influence of induced surface nonuniformity it is instructive to use the surface electronic gas model [30] proposed for accounting the lateral interactions. The derivation of the two-step sequence adhering to the surface electronic gas model was reported previously [31, 32]. The surface electron gas model, not applicable at very low coverage, explains mutual interactions between adsorbed species and the catalyst by changes in the position of the Fermi level. It assumes a complete or partial ionization of 
the adsorbed species taking place during chemisorption with an electron transfer to the surface layer. As shown in [32] the model gives the Fowler-Guggenheim adsorption isotherm, different from the Langmuir isotherm by an exponential term accounting for lateral interactions.

Rearranging an expression presented in [32] for the medium coverage the reaction rate can be written as

$v=\frac{4 \pi m * k_{B} T}{\eta^{2} h^{2}} \ln \left(\frac{\omega_{1}+\omega_{-2}}{\omega_{-1}+\omega_{2}}\right) \frac{\omega_{1} \omega_{2}-\omega_{-1} \omega_{-2}}{\left(\omega_{1}+\omega_{-2}\right)^{\alpha}\left(\omega_{-1}+\omega_{2}\right)^{1-\alpha}}$

where $\omega_{i}$ are frequencies of steps (i.e. $\omega_{+1}=k_{+1} P_{A}$,etc.), and $\eta$ is the effective charge acquired by the adsorbed species, $h$ is the Planck constant, $k_{B}$-Boltzmann constant, m*-effective electron mass.

A simple analysis of the two-step sequence with both irreversible steps gives
In the current treatment it is worth to consider the Langmuir-Hinshelwood mechanism

1. $A+Z \Xi Z A$ (quasi - equilibrium)

2. $B+Z \Xi Z B$ (quasi - equilibrium)

3. $\mathrm{ZA}+\mathrm{ZB} \Rightarrow \mathrm{C}+2 \mathrm{Z}$

$\mathrm{A}+\mathrm{B} \rightarrow \mathrm{C}$

where for simplicity adsorption steps are considered as quasi-equilibria and the surface reaction between the two adsorbed species (i.e. step 3 in Eq. 21) is supposed to be the rate-determining step. The reaction rate is expressed for the irreversible reaction by

$$
v=\frac{k_{3}^{0} K_{1}^{0} K_{2}^{0} P_{A} P_{B} e^{\left(-E_{3}-\Delta H_{1}-\Delta H_{2}\right) / R T}}{\left(1+K_{1}^{0} P_{A} e^{-\Delta H_{1} / R T}+K_{2}^{0} P_{B} e^{-\Delta H_{2} / R T}\right)^{2}}
$$

with the value of an apparent activation energy equal to

$$
E_{a c t}=(1-\alpha) E_{1}+\alpha E_{2}
$$

The value of the Polanyi parameter is often within $0.3 \div 0.7$, with a typical value of 0.5 . Analysis of Eq. (20) shows that for the case of lateral interactions when the reaction proceeds in the range of medium coverage (for example ca. $0.2 \div 0.8)$ the apparent activation energy is a mean value of the activation energies of elementary steps independent on the pre-exponential factors.

\section{Langmuir-Hinshelwood Mechanism with a Non-linear Step}

The scheme discussed above contains only linear steps, i.e. only one (either free or an occupied) site is present on the left or right sides of equations for elementary reactions. In case of nonlinear steps due to nonlinearity, the resulting equations can be difficult to solve and can lead to multiple steady states [33]. The reaction mechanisms cannot be presented using a simple concept of catalytic cycles [34] and the topological representation of catalytic cycles with nonlinear steps requires that the nodes comprise all possible surface species including free sites while branches indicate interconnections between reactions. where $K_{A}^{0}$ and $K_{B}^{0}$ denote pre-exponential factors of adsorption constants of A and B. At low coverage of reactants both terms in the denominator of Eq. (22) are smaller than unity thus $E_{a c t}=E_{3}+\Delta H_{1}+\Delta H_{2}$. On the contrary at high surface coverage of a certain compound (e.g. A) the respective term in the denominator is larger than other terms (reflecting the coverage of the other compound and the fraction of vacant sites), finally giving an expression for the apparent activation energy $E_{a c t}=E_{3}-\Delta H_{1}+\Delta H_{2}$. This simple example clearly illustrates, similar to an example of a linear mechanism discussed above, that the activation energy depends on the experimental conditions and can change when the reaction order is changing.

Moreover, it can be demonstrated similar to the case of linear reaction mechanism, that the apparent activation energy for a structure sensitive catalytic reaction depends also on the cluster size

$$
v=\frac{k_{3}^{0, t} K_{A}^{0, t} P_{A} K_{B}^{0, t} P_{B} e^{\frac{-\alpha_{3}\left(\Delta \Delta G_{a d s, A}+\Delta \Delta G_{a d s, B}\right) / d_{c l u s t e r}-E_{3}-\Delta H_{1}-\Delta H_{2}}{R T}}}{\left(1+K_{A}^{0, t} P_{A} e^{\frac{-\Delta \Delta G_{a d s, A} / d_{\text {cluster }}-\Delta H_{1}}{R T}}+K_{B}^{0, t} P_{B} e^{\frac{-\Delta \Delta G_{a d s, B} / d_{\text {dcluster }}-\Delta H_{2}}{R T}}\right)^{2}}
$$

with $\Delta \Delta G_{a d s, A}=\left(\Delta G_{a d s, e d g e s, A}-\Delta G_{a d s, \text { terraces }, A}\right)$ reflecting difference in the Gibbs adsorption energy of the substrate A on edges and terraces, etc., and the values of pre-exponential factors corresponding to the reaction on terraces. 
For the domain of low coverage when the structure sensitivity is pronounced, contribution of the terms containing $\Delta \Delta G_{a d s, A}$ and $\Delta \Delta G_{a d s, B}$ cannot be neglected and the apparent activation energy depends also on the cluster size.

Experimental data reporting changes in the activation energy with the cluster size were presented recently for dehydrogenation of propane [35]. The reaction mechanism in a simplified form with the first $\mathrm{C}-\mathrm{H}$ activation steps being the rate limiting can be given as

1. $\mathrm{AH}_{2}+\mathrm{Z} \Xi \mathrm{ZAH}_{2}$ (quasi - equilibrium) 1

2. $\mathrm{H}_{2}+2 \mathrm{Z} \Xi 2 \mathrm{ZH}$ (quasi - equilibrium) -1

3. $\mathrm{ZA}+\mathrm{Z} \Rightarrow \mathrm{ZAH}+\mathrm{ZH}$

$3^{\prime} \cdot \mathrm{ZAH}+\mathrm{Z} \Rightarrow \mathrm{Z}+\mathrm{A}+\mathrm{ZH}$ (fast) $\quad 1$

$\mathrm{AH}_{2} \rightarrow \mathrm{A}+\mathrm{H}_{2}$

where on the right hand of the expressions for elementary steps their stoichiometric numbers [36] are listed. The rate expression for mechanism (24) can be derived in a similar fashion for the Langmuir-Hinshelwood mechanism with dissociation

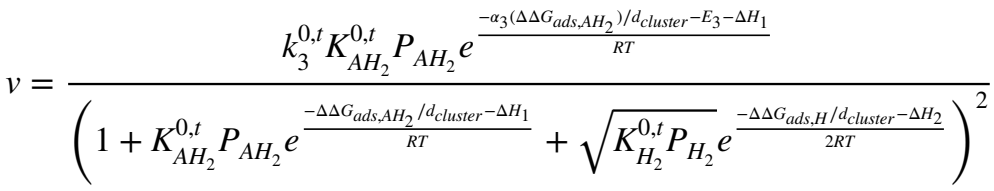

Comparison between experimental data of [35] and Eq. (26) is presented in Fig. 1, illustrating an increase of the apparent activation energy when the size of clusters is increasing in agreement with the theoretical prediction. The value of parameter $\alpha_{3}\left(\Delta \Delta G_{a d s, A H_{2}}\right)$ was calculated as ca. $-77 \mathrm{~kJ} / \mathrm{mol}$, pointing out on a substantial difference between the Gibbs energy of adsorption of the alkane on edges and terraces, while the sum of $E_{3}+\Delta H_{1}$ is ca. $105 \mathrm{~kJ} / \mathrm{mol}$.

In the particular case of propane dehydrogenation, the activation energy is approaching this value of $105 \mathrm{~kJ} / \mathrm{mol}$, while the minimum observed activation energy should correspond to the isolated single atom catalysts. For the case, when there is a small difference between the Gibbs energy of adsorption of the alkane on edges and terraces, the reaction is structure insensitive and the apparent activation energy, as expected, does not change with the cluster size. When $\Delta \Delta G_{a d s}$ for adsorption of an alkane is positive, the apparent activation energy on smaller clusters is higher than for larger clusters levelling off upon the cluster size increase.

Typically limited experimental data are available for
In the case when the observed reaction order in propane is equal to unity and the order in hydrogen is one, the activation energy is just

$E_{\text {act }}=\frac{\alpha_{3}\left(\Delta \Delta G_{a d s, A H_{2}}\right)}{d_{\text {cluster }}}+E_{3}+\Delta H_{1}$

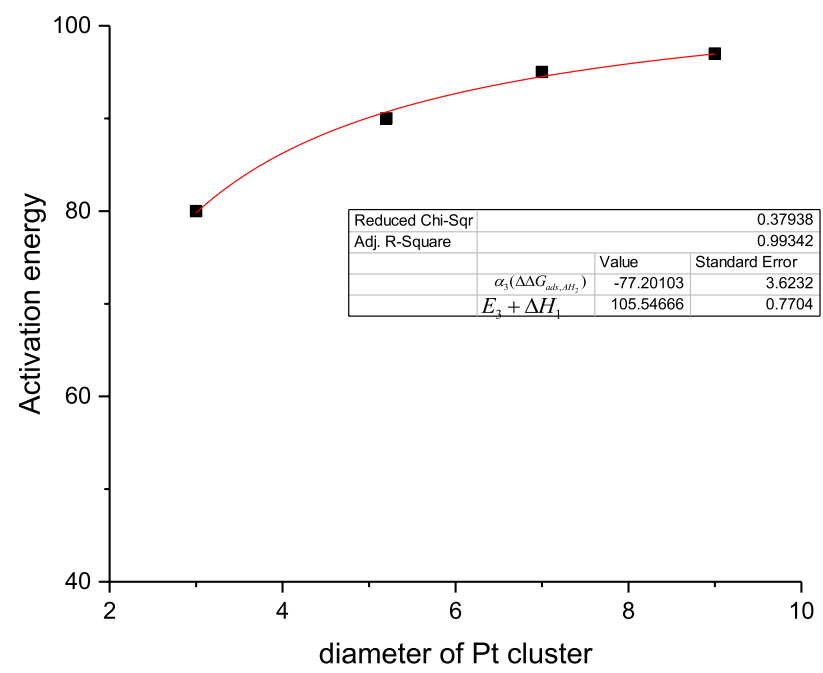

Fig. 1 Activation energy in propane dehydrogenation as a function of cluster size. Comparison between experimental [35] and calculations according to Eq. (26) temperature dependent structure sensitive reactions, when also a reliable kinetic model for the corresponding reaction is established. The current work provides a mathematical framework for analysis of apparent activation energy in the case of such structure sensitive reactions and hopefully will serve a starting point for kinetic studies of such sensitive reactions, when determination of the apparent activation energy for catalysts bearing different cluster sizes will be combined with development of a reliable kinetic model. Such combination, comprising also theoretical and experimental elucidation of the Gibbs energy of adsorption on edges and terraces, will allow to address how physically reasonable are the parameters, which follow the analysis of the apparent activation energy dependence on the cluster size.

\section{Deactivation}

In the treatment above only intrinsic activity was considered as a descriptor of the catalyst performance. While activity and selectivity are often the primary concerns of research in academia [37], long term stability and deactivation are usually the least explored. This treatment is focused on the apparent activation energy for some complex reaction, thus it would be interesting to analyse what would be the impact 
of deactivation on apparent activation energy for structure sensitive reactions.

The theoretical treatment was presented in [38] for the case of two step sequence when coking occurs as a result of the reactant A interacting with an intermediate I. The following expression was obtained for the steady state rate in the case of separable kinetics, when deactivation does not depend on the kinetic parameters of the main reaction

$r_{\text {deact }}=\frac{r_{0}}{K_{K}^{\prime} e^{\frac{-\chi_{K}}{d_{\text {chucter }}}} C_{A}+1}$

where $r_{0}$ is the initial rate defined through the apparent rate constant, reaction order and activation energy as $r_{0}=k_{a p p}^{0} C_{A}^{n} \exp \left(\frac{-E_{a p p, 0}}{R T}\right), r_{\text {deact }}$ is the rate when the catalyst deactivates $r_{\text {deact }}=k_{\text {app,deact }}^{0} C_{A}^{n \text {,deact }} e^{\frac{-E_{\text {app,deact }}}{R T}} ; K_{K}^{\prime}$ is the ratio between coking and self-regeneration constants, $\chi_{K}$ reflects the difference between the Gibbs energy of adsorption of coke on edges and terraces $\chi_{K}=\left(\Delta G_{K, \text { edges }}-\Delta G_{K, \text { terraces }}\right) / R T$ and $C_{A}$ is the concentration of reactant $\mathrm{A}$.

Such formulation allows to consider cases when, for example, edge sites, being intrinsically more reactive with higher rate constants, are more severely poisoned or inactivated by coke because of a stronger binding of coke or stronger poisoning.

In the continuous approach adapted above (Eq. 10) affinities of the terraces and edges are represented by different values of the Gibbs energy of adsorption on these sites. Reactivity in the main reaction was thus expressed through $\chi$, which reflects such difference between Gibbs energy of adsorption on edges and terraces. Different level of poisoning by edges and terraces is considered in the current model in a similar way by introducing the parameter $\chi_{K}$ accounting for the difference between the Gibbs energy of adsorption of coke on edges and terraces. Different affinity of coke would give the value of parameter $\chi_{K}=\left(\Delta G_{K, \text { edges }}-\Delta G_{K, \text { terraces }}\right) / R T$ deviating from zero and this in turn will influence how deactivation depends on the cluster size.

An alternative to this approach, would be to explicitly consider the overall rate as the sum of contributions on different types of sites with varying coverage when the cluster size is changing. Such changes in coverage follow implicitly from Eq. (7) as the values of rate constants (or adsorption coefficients for other reaction mechanisms) are different on edges, corners and terraces.

It was demonstrated in [28] for the deactivation free reaction that when TOF is either increasing or decreasing with the cluster size both models (i.e. discrete and continuous) give equivalent description of the experimental data. The same can be expected for structure sensitive deactivation.
Within the framework of the continuous approach adapted in the current work, the initial rates in deactivation free conditions and for the deactivated catalyst can be described using power low expressions with temperature dependent apparent rate constants, thus giving

$k_{\text {app,deact }}^{0} C_{A}^{n, \text { deact }} e^{\frac{-E_{\text {app,deact }}}{R T}}=\frac{k_{\text {app }}^{0} C_{A}^{n} e^{\frac{-E_{\text {app }, 0}}{R T}}}{1+K_{K}^{0} C_{A} e^{\frac{-\Delta \Delta G_{\text {ads.coke }} / d_{\text {cluster }}-\Delta H_{K}}{R T}}}$

In case of strong deactivation, the second term in denominator of Eq. (28) prevails and subsequently one gets

$E_{\text {act }, \text { deact }}-E_{\text {act }, 0}=\frac{\left(\Delta \Delta G_{a d s, K}\right)}{d_{\text {cluster }}}+\Delta H_{k}$

where $\Delta \Delta G_{a d s, K}=\left(\Delta G_{\text {ads,edges }, K}-\Delta G_{\text {ads,terraces }, K}\right)$ reflects in the difference in the Gibbs energy of adsorption of coke on edges and terraces.

Difference between the apparent activation energies for the deactivated catalyst and in the deactivation free conditions thus depends inversely on the cluster size. The enthalpy of coking is positive considering the endothermic nature of reactions leading to deactivation (dehydrogenation, cracking). The absolute value of $E_{\text {act,deact }}-E_{a c t, 0}$ can be either positive or negative depending thus on $\Delta G_{K, \text { edges }}-\Delta G_{K, \text { terraces }}$. Within the framework of the theoretical approach discussed above a stronger Gibbs adsorption energy on terraces than on edges will result in a larger difference between the apparent activation energy on smaller

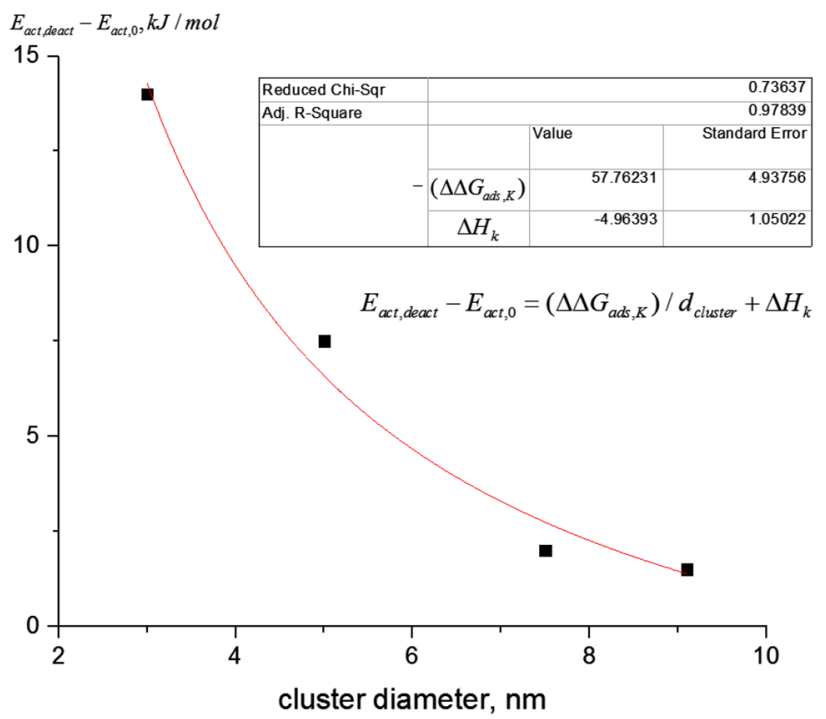

Fig. 2 Difference between activation energy for fresh and deactivated catalysts as a function of platinum cluster size. Comparison between experimental [35] data on propane deactivation and calculations according to Eq. 29 
clusters in line with experimental data of [35] on propane dehydrogenation on Pt. Figure 2 illustrates that Eq. (29) is able to quantitatively describe the difference between apparent activation energies of the fresh and deactivated catalysts. The calculated value of $\Delta H_{K}$ is rather low requiring a further theoretical analysis, which is outside of the scope of the current contribution.

The theoretical treatment in [38] for the two step sequence considered not only coking, but also poisoning assuming that a free unoccupied site is required for adsorption of a poison, which in a general case can be reversible. In the case of non-separable kinetics the rate expression for a deactivating catalyst as a function of time on stream was derived [38];
$W=\frac{k_{+1}^{0} P_{A} e^{-\alpha \chi / d_{c l}}+k_{-1}^{0} P_{C} e^{(1-\alpha) \chi / d_{c l}}}{k_{+2}^{0} P_{B} e^{(1-\alpha) \chi / d_{c l}}}$

where $\chi$ is given by Eq. (9). For deactivation at infinite time when a stationary state is reached, expression (34) can be simplified

$r_{\text {deact }}=\frac{r_{0}}{\frac{K_{p}^{\prime} e^{\frac{-\chi_{p}}{d_{c l}}} C_{P}}{W+1}+1}$

allowing to analyse the apparent activation energy in a similar was as it was done for coking

$r_{\text {deact }}=r_{0}\left[\frac{k_{-p}(W+1)}{k_{+p} C_{P}+k_{-p}(W+1)}+\frac{k_{+p} C_{P}}{k_{+p} C_{P}+k_{-p}(W+1)} e^{-\frac{k_{+p} C_{P}+k_{-p}(W+1)}{1+W} t}\right]$

where $C_{p}$ is the concentration of the poison, $k_{+p}$ and $k_{+p}$ are the kinetic constants of poisoning in the forward and reverse direction, respectively and $\mathrm{W}$ is given by

$W=\frac{k_{+1} P_{A}+k_{-1} P_{C}}{k_{+2} P_{B}+k_{-2} P_{D}}$

In [38] it was assumed that $1+\mathrm{W} \approx 1$ giving an expression for separable kinetics, when deactivation is completely independent on the main reaction. In a more general case

$r_{\text {deact }}=r_{0}\left[\frac{1}{\frac{K_{p} C_{P}}{W+1}+1}+\frac{1}{1+\frac{(W+1)}{K_{p} C_{P}}} e^{-\left(k_{+p} C_{P} \frac{1}{1+W}+k_{-p}\right) t}\right]$

The rate constants for poisoning are

$k_{+p}=k_{+p}^{\prime} e^{\frac{-\alpha_{p} \chi_{p}}{d_{c l}}} ; k_{-p}=k_{-p}^{\prime} e^{\frac{\left(1-\alpha_{p}\right) x_{p}}{d_{c l}}}$

where $\alpha_{\mathrm{p}}$ is the Polanyi parameter for the poisoning step and $\chi_{p}=\left(\Delta G_{p, \text { edges }}-\Delta G_{p, \text { terraces }}\right) / R T$, reflecting the difference between the Gibbs energy of adsorption of the poison on edges and terraces.

The time on stream dependence of the reaction rate in the case of poisoning is thus

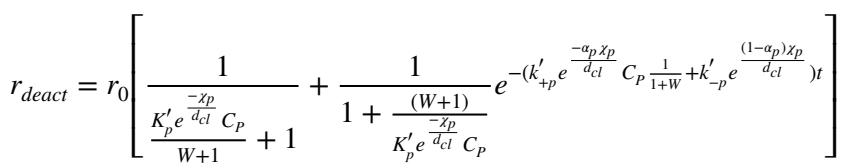

For a specific case of the second step in mechanism (2) being irreversible and the same values for Polanyi parameter in both steps a general expression for $\mathrm{W}$ given in [38] is reduced to

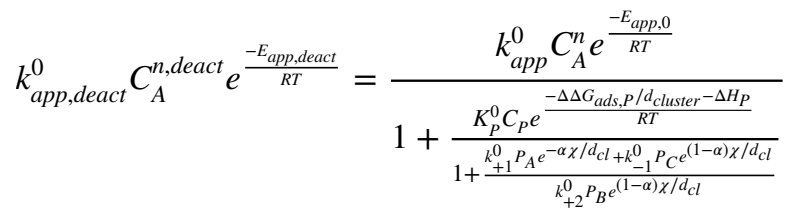

The value of the apparent activation energy has thus a rather complex dependence on the cluster size.

For the case of separable poisoning Eq. (37) is transformed into

$$
k_{\text {app,deact }}^{0} C_{A}^{n, \text { deact }} e^{\frac{-E_{\text {app,deact }}}{R T}}=\frac{k_{a p p}^{0} C_{A}^{n} e^{\frac{-E_{a p p, 0}}{R T}}}{1+K_{P}^{0} C_{P} e^{\frac{-\Delta \Delta G_{a d s, P} / d_{\text {dluster }}-\Delta H_{P}}{R T}}}
$$

which is conceptually very similar to the case of coking discussed above.

\section{Conclusion}

Interpretation of catalytic kinetics is quite often simplified by considering turnover frequencies as a measure to quantify performance of catalysts. Moreover, power-law kinetics with fractional orders to reactants and an apparent constant with an exponential temperature dependence are often utilized as a proxy for reaction kinetics based on a step-wise reaction mechanism.

Evaluation of the reactions orders by linearizing experimental data and plotting logarithms of rates vs logarithm of concentrations is very straightforward. When the orders in the reactants are fractional it is often concluded that a particular reaction is a complex one comprising several steps. Such conclusion about a multistep reaction might be 
hindered when only a simplified concept of an apparent activation energy is applied. In particular, the current theoretical analysis shows that interpretation of the apparent activation energy is far from being trivial in the case of complex structure sensitive catalytic reactions with several steps. For a two step sequence when only the second step determines the overall rate, the activation energy can either increase or decrease as a function of the cluster size depending on which types of sites the Gibbs energy of adsorption is larger. For the same reaction sequence with catalyst deactivation by coking originating from the intermediate, there could be a difference between the apparent activation energies for the deactivated catalyst and in the deactivation free conditions depending inversely on the cluster size.

Acknowledgements Open access funding provided by Abo Akademi University (ABO).

\section{Compliance with Ethical Standards}

Conflict of interest The author declares no conflict of interest.

Open Access This article is distributed under the terms of the Creative Commons Attribution 4.0 International License (http://creativeco mmons.org/licenses/by/4.0/), which permits unrestricted use, distribution, and reproduction in any medium, provided you give appropriate credit to the original author(s) and the source, provide a link to the Creative Commons license, and indicate if changes were made.

\section{References}

1. Tsukamoto M, Gopalaiah K, Kagan HB (2008) Equilibrium of homochiral oligomerization of a mixture of enantiomers. Its relevance to nonlinear effects in asymmetric catalysis. J Phys Chem 112:15361-15368

2. Blackmond DG (2015) Kinetic profiling of catalytic organic reactions as a mechanistic tool. J Am Chem Soc 137:10852-10866

3. Helfferich FG (2001) Kinetics of homogeneous multistep reactions. In: Compton RG, Hancock G (eds) Comprehensive chemical kinetics, vol 38. Elsevier, Amsterdam

4. Leskovac V (2003) Comprehensive enzyme kinetics. Kluwer Academic/Plenum Publishers, New York

5. Temkin ON (2012) Homogeneous catalysis with metal complexes: kinetic aspects and mechanisms. Wiley, Weinheim

6. Murzin DY, Salmi T (2016) Catalytic kinetics, chemistry and engineering, 2nd edn. Elsevier, New York

7. Salmi TO, Mikkola J-P, Wärnå J (2010) Chemical reaction engineering and reactor technology. CRC Press, Boca Raton

8. Fogler HS (1998) Elements of chemical reaction engineering, 3d edn. Prentice Hall, NJ

9. Marin G, Yablonsky GS (2011) Kinetics of chemical reactions. Wiley, New York

10. Kapteijn F, Berger RJ, Moulijn JA (2008) Rate procurement and kinetic modelling, handbook of heterogeneous catalysis. DOI: https://doi.org/10.1002/9783527610044.hetcat0092. Wiley, New York

11. Bures J (2016) A simple graphical method to determine the order in catalyst. Angew Chem 128:2068-2071
12. Bures J (2016) Variable time normalization analysis: general graphical elucidation of reaction orders from concentration profiles. Angew Chem Intern Edit 55:16084-16087

13. Konsler RG, Karl J, Jacobsen EN (1998) Cooperative asymmetric catalysis with dimeric salen complexes. J Am Chem Soc 120:10780-10781

14. Rosner T, Le Bars J, Pfaltz A, Blackmond DG (1998) Kinetic studies of Heck coupling reactions using palladacycle catalysts: experimental and kinetic modeling of the role of dimer species. $\mathrm{J}$ Am Chem Soc 123:1848-1855

15. Noble-Teran ME, Buhse T, Cruz J-M, Coudret C, Micheau J-C (2016) Nonlinear effects in asymmetric synthesis: a practical tool for the discrimination between monomer and dimer catalysis. ChemCatChem 8:1836-1845

16. Temkin MI (1979) The kinetics of some industrial heterogeneous catalytic reactions. Adv Catal 28:173-291

17. Boudart M, Tamaru K (1991) The step that determines the rate of a single catalytic cycle. Catal Lett 9:15-22

18. Temkin M (1935) Acta Physicochim URSS. 2:313-315

19. Marin GB, Yablonsky GS, Constales D (2019) Kinetics of chemical reactions: decoding complexity, 2nd edn. Wiley, New York

20. Boudart M (1969) Catalysis by supported metals. Adv Catal 20:153-166

21. van Santen RA (2009) Complimentary structure sensitivity and insensitive catalytic relationships. Acc Chem Res 42:57-66

22. Murzin DY (2010) Kinetic analysis of cluster size dependent activity and selectivity. J Catal. 276:85-91

23. Murzin DY (2011) Nanokinetics for nanocatalysis. Catal Sci Tech. $1: 380-384$

24. Murzin DY (2012) On cluster size dependent activity and selectivity in heterogeneous catalysts. Catal Lett. 142:1279-1285

25. Ligthart DAJM, van Santen RA, Hensen EJM (2011) Influence of particle size on the activity and stability in steam methane reforming of supported Rh nanoparticles. J Catal 280:206-220

26. Jones G, Jakobsen JG, Shim SS, Kleis J, Andersson M, Rossmeisl J, Bligaard T, Helveg S, Hinnemann B, Rostrup-Nielsen JR, Chorkendorff I, Sehested J, Nørskov JK (2008) First principles calculations and experimental insight into methane steam reforming over transition metal catalysts. J Catal 259:147-160

27. Kannisto H, Arve K, Pingel T, Hellman A, Härelind H, Eränen K, Olson E, Skoglundh M, Murzin DY (2013) On the performance of $\mathrm{Ag} / \mathrm{Al}_{2} \mathrm{O}_{3}$ as $\mathrm{HC}-\mathrm{SCR}$ catalyst-influence of silver loading, morphology and nature of the reductant. Catal Sci Tech 3:644-653

28. van Hardeveld R, Hartog F (1969) The statistics of surface atoms and surface sites on metal crystals. Surf Sci 15:189-230

29. Arnaut L, Formosinho S, Burrows H (2006) Chemical kinetics, from molecular structure to chemical reactivity. Elsevier, Amsterdam

30. Temkin MI (1972) Theoretical models of the kinetics of heterogeneous catalytic reactions. Kinet Katal 13:555-565

31. Murzin DY (1993) Structure insensitivity-application of the surface electronic gas model. Catal Lett. 22:157-164

32. Murzin DY (1995) Modeling of adsorption and kinetics in catalysis over induced nonuniform surfaces: surface electronic gas model. Ind Eng Chem Res. 34:1208-1218

33. Murzin DY (2017) Interpretation of rate optima vs reaction parameters in steady state catalytic kinetics: molecular aspects beyond concentration dependences. Mol Catal. 433:321-333

34. Murzin DY (2007) On topological representation of catalytic cycles with nonlinear steps. React Kinet Catal Lett. 90:225-232

35. Zhu J, Yang M-L, Yu Y, Zhu Y-A, Sui Z-J, Zhou X-G, Holmen A, Chen D (2015) Size dependent reaction mechanism and kinetics for propane dehydrogenation over Pt catalysts. ACS Catal 5:6310-6319 
36. Horiuti J, Nakamura T (1967) On the theory of heterogeneous catalysis. Adv Catal 17:1-74

37. Scott SL (2018) A matter of life (time) and death. ACS Catal 8:8597-8599
38. Murzin DY (2014) Catalyst deactivation and structure sensitivity. Catal Sci Tech. 4:3340-3350

Publisher's Note Springer Nature remains neutral with regard to jurisdictional claims in published maps and institutional affiliations. 